\section{Research Article}

(C) 2021 Jaber et.al..

This is an open access article licensed under the Creative Commons Attribution-NonCommercial 4.o International License (https://creativecommons.org/licenses/by-nc/4.o/)

\title{
Reflections on Protolanguage: Evidence from Studies on Animal Communication
}

\author{
Aziz Jaber \\ Osama Omari \\ Mujdey Abudalbuh \\ Department of English Language and Literature, \\ Yarmouk University, Shafiq Irshidat st., Irbid, Jordan
}

DOI: https://doi.org/10.36941/jesr-2021-0o12

\section{Abstract}

The paper is a critique of the existence of protolanguage based on some personal reasoning given the findings of previous research. This paper focuses on the nature of semantic compositionality and its existence (and therefore manifestations) in animal communication systems as evidence of the existence of protolanguage. This compositional state that started to color human language has paved the way to hierarchical syntax and thus has helped in the emergence of a recursive, fully complex language. On the other hand, non-human animal communication systems, including those examined in this paper, have not managed to progress beyond the holophrastic state, and thus remained highly confined and unproductive. This is explicated by the observation that these systems do not employ discrete meaningful units that can be used recursively in different linguistic contexts, a necessary condition for a system of communication to be compositional. This is interesting in the study of language evolution as it could suggest that human language could not have evolved from a rudimentary, intermediate stage called protolanguage. Speculating about the existence of protolanguage subsumes convergent evolution (e.g. songbirds). The lack of semantic compositionality in non-human communication system suggests that convergent evolution is hard to prove, which puts the existence of protolanguage on the line. This thesis is, however, far from being established and requires a lot of further research to prove its validity.

Keywords: compositionality, animal communication, protolanguage, language evolution

\section{Introduction}

Recently, language origin (and evolution) has been a hot topic among both linguists and scientists interested in solving the puzzle of the development of the most influential invention in human history, language. Language, as a cognitive niche, lays the basis for human survival and development. In the field of Evolution of Language, researchers use various approaches in an attempt to explain how our species moved from a wholly alanguage state to the complete possession of the complex language we have in modern times. This ambitious endeavor is fraught with two essential inherent problems: (1) the time period involved in language evolution studies is vast; (2) there is a lack of physical evidence 
for it since language leaves no fossilized traces.

Taking these two crucial limitations into account, researchers split into two camps in their pondering over the origin of language, (see section 2). The first viewpoint (e.g. Szathmáry and Maynard Smith 1995; Pinker 2003; Bickerton 2002, 2007; Arbib 2002, 2017; Tallerman 2016,2017) assumes a stable intermediary linguistic state supposed to bridge the gap between no-language state of our ancestors and full language state of modern times, called protolanguage. The other alternative viewpoint (e.g. Buss et al. 1998; Hauser et al. 2002; Berwick et al. 2013; Bolhuis et al. 2014; Nóbrega, and Miyagawa, 2015, 2018, Chomsky 2017) rejects the adaptation hypothesis and instead proposes an all-at-once evolution as a by-product of other evolutionary development.

The paper is a critique of the existence of protolanguage based on some personal reasoning given the findings of previous research. it focuses on the nature of semantic compositionality and its existence (and therefore manifestations) in animal communication systems as evidence of the existence of protolanguage. This compositional state that started to color human language has paved the way to hierarchical syntax and thus has helped in the emergence of a recursive, fully complex language.

This paper is organized as follows. We will begin in section (2) by presenting a short background of the above competing viewpoints about language origin and evolution. In section (3) we will critically review the findings of some major studies on semantic compositionality in non-human animal communication systems. We will then regress in section (4) to a discussion of semantic compositionality, demonstrating its complexity in modern human language. Section (5) presents the conclusions and limitations of this critique paper.

\section{Alanguage State to Full Language State}

Speculating about the problem of evolution of language first arose in the mid-twentieth century after a long lapse (Chomsky 2017). Linguists and other interested scholars adopted two different views as to how language evolved. The first viewpoint approximates Darwin's evolution and natural selection in dealing with human language evolution as a kind of communication-driven adaptation. This adaptation shaped language into its current state as a system of many-folded complexity able to produce, through a finite set of words coupled with a rather small set of rules that pattern them, an infinite number of sentences, familiar and novel, at the expense of the speakers to communicate whatever thought about the world they wish to communicate.

Proponents of this view think that humans did not move from a language-free state to a full language state in one single mutation or innovation. Instead, there is a shared belief that an intermediary phase in language evolution bridged the gap between our non-linguistic ancestors, with a very limited communication system based entirely on innately driven vocalizations, and our linguistic species, enjoying the full complex productive language, primarily learned. These scholars agreed to term this transitional stage "protolanguage" (Smith 2006, 2008). In an influential study, Jackendoff (2011,614-615) defines the topography of this language-sparking phase in human history as:

A system in which words can be concatenated indefinitely, but in the manner of a shopping list (i.e. a ' $W^{* \prime}$ grammar). A protolanguage has phonology, but it has little or no constituent structure, no recursion, no subordination, and no functional categories. The semantic relations among words are signaled by word order alone, using default principles such as agent first, focus last, and modifier adjacent to modified. Since all combinatoriality is driven by honology and semantics, there is no need for words to be categorized by parts of speech.

Under this account, a "synthetic" model of protolanguage is presented. According to proponents of this model (e.g. Bickerton 1990, 1995, 2007, 2010; Jackendoff 1999, 2011; Tallerman 2007, 2010, 2017), protolanguage exhibited symbols that can be paired arbitrarily with atomic meanings which can be sequenced in ad hoc manner to convey larger meanings. From this stage, according to this model which exhibits a little grain of grammatical compositionality, developed our full language by the advent of 
syntax at (a) later stage(s). ${ }^{1}$

The other alternative viewpoint (e.g. Buss et al. 1998; Hauser et al. 2002; Berwick et al. 2013; Bolhuis et al. 2014; Nóbrega, and Miyagawa, 2015, 2018, Chomsky 2017) rejects the adaptation hypothesis and instead proposes an all-at-once evolution as a by-product of other evolutionary development. Some language aspects are taken as by-products of language faculty in the narrow sense (FLN) that, according to this approach, might have evolved for functions other than language; number, navigation, and social relations are some of the suggested reasons. Language, according to this viewpoint, is a mere manifestation of some general cognitive abilities, Language Faculty, just like cultural learning or social intelligence (Hauser et al. 2002). Proponents of this model believe that a mutation did cause the development of the system. For them, the obvious claim is that there is no such intermediate stage called protolanguage. They reject the idea that languages evolve; instead, they believe that the faculty of language has evolved but languages only change, (see e.g. Bolhuis et al. 2014 and Chomsky 2017). They believe that "language should be understood as a particular computational cognitive system, implemented neurally, that cannot be equated with an excessively expansive notion of "language as communication"." (Bolhuis et al. 2014: 1).

The two camps, with substantial differences in scope and purposes though, employ the comparative approach to settle the debate about language origin and evolution. Proponents of the adaptation perspective employ the comparative approach that uses data from domains outside of human language (such as animal communication systems) as well as data from outside of the domain of communication altogether. Proponents of the non-adaptation perspective use the comparative method in the quest of language origin to prove the uniqueness of some allegedly human-specific language traits, to judge whether a shared trait is selected for the same selection pressure or not, or reveal regularities in evolution.

The two models, however, consider comparative studies necessary for the study of human language evolution as they believe that some traits of human communication are also shared in animal communication, a state of affairs which could suggest many things: (1)these common features could be a sign that human and non-human communication systems have similar/different origins and evolutions, and (2) the modern animal communication systems might or might not mirror a more primitive stage of human language, (3)how different in quality and quantity the two systems are, and (4) how the available hypotheses about the evolution of the faculty of language are assessed (see Hauser et al., $\mathbf{2 0 0 2}$ for more details on each outcome of applying the comparative method).

\section{Semantic Compositionality in Animal Communication Systems}

\subsection{Animal languages vs. human languages}

The discrepancy of human language from other non-human communication systems derives from the amount of complexity that human languages exhibit over animals' communication systems. Our language may satisfactorily express more factual meanings than those conveyed by any other nonhuman communication system. There is a common belief that "any human language is capable of communicating the sum total of all that any animal species can communicate, and more. More, because we alone, as far as we know, can tell each other about fictional or abstract objects, and about events far distant in time and space" (Hurford 2008, 249). According to Hauser et al. (2002), complexity in our

\footnotetext{
${ }^{1}$ We would like to note that this proposal has not gained unanimous support within the adaptation model of language evolution. A completely opposing model called the "holistic" approach has been proposed. Under this account (Wray 2002a; Arbib 2008; Arbib et al. 2010), protolanguage exhibited holophrastic arbitrarily strung together noncompositional utterances which lack internal morphological structure, conveying complex event-related propositions, rather than semantic atoms. However, discussing the differences between these two versions falls outside the scope of this paper; what is relevant is that both versions assume protolanguage as an intermediary stage.
} 
language springs from a distinction between two types of the language faculty. The first, termed FLB (faculty of language in the broad sense), contains sensory-motor capacities (the phonological system), and conceptual-intentional capacities (the semantic system), such as auditory channels, memory, intelligence, and shared attention. Most of these features are shared by other species. The second one, FLN (faculty of language in the narrow sense), itself a component of FLB, includes capacities restricted to language and other computational operations, represented solely by recursion, attributed to narrow syntax, as the only component in this sphere. The latter, through facilitating recursion, is responsible for the complexity of our language.

Our ability to parse a recursive structure reveals another thought-to-be-unique-to-humanlanguage principle, the principle of compositionality. Whereas, other non-human communication systems are thought to be either holistic-where no subpart of, say, the call or screech denotes part of the whole meaning-or at best combinatorial, "that is, they are made up of several reusable subunits strung together" (Hurford20o8, 254). A clearer distinction between the two mechanisms is substantial here. A compositional mechanism, as defined above, draws upon atomic self-meaningful units that can be structured innovatively, according to some syntactic rules, to create novel meanings in unlimited different situations. A combinatorial mechanism, however, uses identifiable, reusable subunits which themselves carry no meanings (Hurford 2008), to convey very limited situation-bound, pre-identified meaning not derived from the meanings of these atomic subunits. To make this distinction obvious, Hurford draws an analogy of our linguistic system which invests the so-called 'double articulation', which is no different from Hockett's (1960) 'duality of patterning'.

\subsection{Samples of animal communication systems}

Many case studies have been conducted on different animals in the hope of showing a grain of compositionality in their communication systems. In this section, a critical review of some of these studies is presented. It is shown that the animal communication system lacks compositionality, a feature which renders human language unique. This main difference has a bearing on predicting the linguistic status of protolanguage and the evolution topography of modern human language. It also has a bearing on the subsumed interaction between protolanguage and other nonhuman communication systems.

\subsubsection{Wedge-capped capuchin monkeys}

The wedge-capped capuchin monkeys (Cebusolivaceus) produce different types of calls in different social circumstances (Robinson 1984). This species of capuchin monkeys is also able to produce these different types of calls in a compound structure of doublets, triplets and sometimes quadruplets. The compound calls are produced in situations "intermediate between those of the constituent calls" (ibid.,46), a state of affairs which suggests an intermediate internal state. It is important to keep in mind that the combinations of calls, though they may seem akin to compounding words in human language, are the expressions of combinations of internal states that are regarded by some (including Chomsky 1972) to "simply express the animal's motivational state, etc." (Robinson 1984, 47). That is, when the Cebus monkeys experience an internal state that does not have a specific call specified for it (which would be genetically encoded), they are capable of expressing "intergradations or intermediates" between these call types using the compounded calls.

The ordering of these compound calls is predictable, and they are 'syntactically' structured. Following Robinson (1984), we refer to this structure as 'syntax'. This syntactic structure is not the same as the human syntax, which is demystified with "organized relations among separate sounds and novel use of different combinations of sounds," but instead consists of a finite number of signals that "do not symbolize or refer to objects in the external environment" and cannot be syntactically organized in the same way humans use syntax (Robinson 1984, 47). The syntax here, Robinson emphasizes, is a "transformational rule that generates a sequence of calls" (P. 72). 
According to Robinson, if we are to properly analyze the syntax of animal communication systems, we must include: “(a) Accurate classification of types of calls. (b) Description of the syntactic rules generating compound calls or call sequences. (c) Examination of the social circumstances or contexts in which both single and compound calls occur" (P. 75). Robinson succeeds in showing these steps in his discussion of a large sample of Cebus calls, probably making the findings and implications of his research more reliable than those of, for instance, the research on the gray parrot discussed below.

Taking this definition of syntax, the syntactically structured compound calls are not uncommon for the capuchin monkeys - around 38\% of the total sample in Robinson's (1984) study. There is a duality of patterning seen in the syntax of Cebus vocalizations $\neg$ - syllables are combined to form calls made in specific circumstances. These calls can then be affixed into compound calls in intermediate situations. However, Robinson finds that there was "no evidence of syntactic rules in Cebus vocal communications analogous to the grammatical rules of human language" (P. 75). The findings suggest that the syntactic rules used to form compound calls in the Cebus monkeys behave like the morphological rules of human languages that combine two lexemes into a new compound. However, their syntactic rules are not analogous to the syntactic rules responsible for generating full sentences in human language.

\subsubsection{Campbell and Diana Monkeys}

Klaus Zuberbühler (2005) reports a non-human communication so close to the compositional communication entertained in human language. The communication involves a response of one ape species, Diana monkey, to another different species, Campbell monkeys. Campbell monkeys employ unitary different calls for leopards and eagles. In non-dangerous situations, Campbell monkeys start their calls with a thirty-second-lasting 'boom' but produce boom-less calls when they feel real imminent danger. Diana monkeys interpret these different calls appropriately, reacting with less panic for the 'boomed' calls, and with more panic for the 'boom-less' ones. What this means is that “... adding 'booms' before the alarm call series of a Campbell's monkey created a structurally more complex utterance with a different meaning than that of alarm calls alone." (ibid.,279). However, Zuberbühler is explicit about the limitedness of these combinatorial calls, observing that none of these apes (or any other ones that produce sequenced calls) can innovate new call types for new situations, or could creatively combine their calls to come up with new novel meanings. According to Jackendoff (2002, 293), the major difference between the best nonhuman combinatorial communication systems and the simplest representation of human language, found in baby talk, for instance, is the non-situationspecificity of human words. The word kitty may be uttered by a baby to draw attention to a cat, to inquire about the whereabouts of a cat, to summon the cat, to remark that something resembles a cat, and so forth. Other primates' calls do not have this property. A food call is used when food is discovered (or imminently anticipated) but not to suggest that food be sought. A leopard alarm call can report the sighting of a leopard but cannot be used to ask if anyone has seen a leopard lately.

\subsubsection{Black-and-white colobus monkeys}

Schel et al. (2009) take on a new proposal from investigating and analyzing alarm calling in two species of black-and-white colobus monkeys, King colobus of Tai Forest, Ivory Coast and Guerezacolobus of Budongo Forest, Uganda. Instead of the acoustically discrete calls elicited by distinct predators, these two species invest combinations of two calls, roaring phrases and snorts, which are relatively acoustically similar, but significantly different in structure, elicited in response to specific predators, crowned eagles, leopards, and sometimes chimpanzees and humans.

The data were collected in two different sites through playing back growls of leopard and shrieks of eagles and recording the vocalizations of monkeys in response to these stimuli. The analysis of the vocal responses was conducted according to six structural parameters: duration of a vocal response, 
the total number of roaring sequences, the total number of roaring phrases in each sequence, the mean number of roaring phrases in each roaring sequence, the number of snorts, and the number of bouts. It was noticed that individual roaring phrases were rare, but rather a sequence of roaring phrases was often produced. This kind of high-pitched call, audible from a distance, was produced in response to calls of other males and/or species, or response to major disturbances, such as falling trees or the presence of predators. The other less audible, low-pitched call, the snort, was given as an alarming call for the presence of a ground predator like leopards, chimps, or humans. Unlike roaring phrases, snorts could be produced alone, but usually before roaring sequences.

Observations in both sites indicated monkeys responding to eagle and leopard playbacks differently. As for leopard playbacks, monkeys predominantly reacted with one bout of roaring, consisting of many sequences with one or two roaring phrases each. The opposite is elicited by eagle playbacks; the monkeys produced one roaring bout containing only two sequences which consisted of a large number of roaring phrases per se.

Interestingly, except for the first and the final roaring phrases, no significant acoustic differences were detected in roaring phrases for both predator types in both species. This suggested that alarm calls employed by these two species were acoustically indistinct for different predators. However, a significant difference in the compositional structure of these roaring sequences was observed in response to eagles and leopards. Only roaring sequences to leopards were preceded by snorts; a higher number of snorts was produced in response to leopard growls than to eagle shrieks, and snorts were produced to other ground predators like chimpanzees and humans.

It could be inferred that the two acoustically different alarm calls were not used separately to one predator class, but instead, being assembled into longer sequences as a way of labeling different predators. In addition, the rate and number of snorts indicated how close or distant leopards were. Vocal responses to leopard and eagles triggered other male monkeys to rush toward the source of the stimulus either to attack the eagle in a group or to closely monitor the leopard to take suitable action. This suggested that black-and-white colobus monkeys, in response to specific predators, produced specific call combinations, rather than single distinct calls. The big question that Schel et al. (2009) alluded to but did not explicitly address was whether such combinations form a step out of the onedistinct-call-one-distinct-predator correspondence, which stamps non-human communication systems, towards the threshold of complex compositionality that distinguishes human language. Or that they are mere nonproductive ways of alarm call attributed to the very few acoustically distinct calls that these monkeys physiologically capable of producing, and hence making use of repeating them in different meaningful sequences.

Though longer series calls provide more information, there is little reason to suggest that these monkeys produce and perceive these combinatorial calls as the sum of the meanings of each component unit in the string ${ }^{2}$. This impression is strengthened by the observation that these strung calls are unproductive, not to mention their event-relatedness as a biological response instigated by the presence of danger. These calls according to Hurford $(2008,254)$ are best termed as "'song' ..., as such complex animal calls are more like music than human language, which expresses semantic content through the application of compositionality."

\subsubsection{The gray parrot}

In the discussions available to-date about animal communication systems, it is often reported that many species of animals, including some mammals, can "categorically distinguish various bits of human speech, for instance cleanly separating speech tokens such as /b/-/p/" (Pepperberg, 2011).

${ }^{2}$ This is merely a reasoning that awaits falsification by conducting more studies on the species. At this point we do not find a more satisfactory justification, so we are not quite sure if this is really the case. 
However, very few species are able to produce human speech with human-like formants. The gray parrot (Psittacuserithacus), which has been long researched for its cognitive and communicative abilities, is one species that has this ability; it has been shown to be able to "acquire and use English speech in ways that often resemble those of very young children" (Pepperberg 2006, 77). Pepperberg's own gray parrot, Alex, was able to "identify, request, refuse, categorize and quantify about 100 different objects" (P. 78). It was also found that Alex was able to combine them in order to perform different semantic functions. This could be interpreted as evidence for semantic compositionality (or combinatoriality), once assumed to exist only in the communication of humans and non-human primates (Pepperberg 2002, 54).

It is noteworthy, though, that Alex's labels are not properly embedded syntactically or semantically, and thus cannot be considered intentional communication (Pepperberg 1999). Pepperberg's findings do have interesting implications for the study of language evolution and the evolution of intelligence, however.

\subsubsection{Carolina Chickadees (Poecilecarolinensis)}

In a pilot study on the chick-a-dee calls of Carolina Chickadees (Poecilecarolinensis), Freebergand Lucas (2002) maintains that the calls of this avian species are structurally complex, exhibiting a set of simple rules that could show how the order of the notes is set. The combining of a varied number of notes creates strings of unique chickadee calls that might be associated with possibly different meanings. It has been found, for example, that Carolina chickadees, upon their first finding of a novel seed source, produce a call type with relatively large numbers of ' $C$ ' notes, relatively lower-pitched notes with a fairly complex and noisy structure. By flying in a flock to the source of the call, the receivers have also been found to respond more promptly to the source of playbacks of calls containing ' $\mathrm{C}$ ' notes than to chickadee calls not containing ' $C$ ' notes. This asymmetry in the response indicates that "receivers respond differently to chick-a-dee calls containing different compositions of note types and represent the first step to link variation in note composition and ordering in these calls to possible meanings" (ibid., 837). As the elementary notes carry no meanings and the final call combinations serve functionally-limited and genetically-endowed stimuli, like finding a novel source of seeds, there is no sense in concluding that the Carolina chickadee's combinatorial calling system exhibits compositional semantics identical with that of the human language. The authors assert, "[w]hereas it has been hypothesized that chick-a-dee calls with different notes may convey different information, no experimental evidence has been offered in support of the hypothesis" (ibid.,837).

\subsubsection{Honeybees}

Honeybees waggle dancing, however, presents itself as compositional, in that the whole meaning of the dance is a function by which atomic meanings combine to yield more complex meaning. Interestingly, each atomic part of the dancing carries meaning: the orientation indicates the angle relative to the sun position; the speed of waggling conveys the distance of the nectar source from the honeycomb; and the duration of the dance relates to the quality of the food source. Distance and angle are necessary for locating the food source, and quality is important for not wasting the bees' precious time and effort (Hurford20o8). The meanings of these three aspects of dancing combine to compose a piece of information like 'There is nectar of 'such' quality at a distance of 'such- time' of flying, to the 'such' direction of the sun'. However, honeybees waggle dancing, though looks compositional in that the elementary components have meanings themselves and their concatenation yields in a bigger meaningful combination; it is still innate and combinatorial. These three subunits are being used in a limited way as a functional event-related, genetically endowed mechanism that repeats itself, without any innovation. To qualify as compositional, the dance subunits must be employed innovatively in contexts other than this one-to-one behavioral response. Bees have never been observed discussing, proposing, planning, or commenting on nectar foraging. 
The above-previewed studies, very limited in number though, do not support the adaptationdriven hypothesis of language evolution. This tentative finding of compositionality in animal communication puts protolanguage on the line because convergence, which is a crucial tool for speculating the existence of an intermediary stage in the comparative analysis of language evolution, does not support its very existence. A big stumbling block for the hypothesis of protolanguage is that, so far, there is no evidence for human-like compositionality in non-human communication systems. There is no obvious reason, however, why such a combinatorial computational system has not evolved in non-human communication systems (Bolhuis 2014). It could have evolved through common descent or convergent evolution if Language is an adaptable, evolutionary system of communication. What seems more plausible is that the faculty of language, rather than its manifestations (human languages), has undergone evolution (Chomsky 2017). Therefore, this study gives some kind of support to the onesingle mutation evolution which led to the emergence of the faculty of language 70,000-100,000 years ago and has not undergone any evolutionary modification since then (Bolhuis 2014).

\section{Semantic Compositionality in Modern Human Language}

Language is a system for encoding and expressing thought (Berwick et al. 2013). However, the big question that has drawn the interest of philosophers and semanticists for centuries is how language encodes thought. The naïve, but apparently wrong, answer is a set of memorized symbols paired with their meanings. This answer is untenable as it cannot account for an intriguing property of human language: 'productivity' (see Hockett 1960). Partee (1995) emphasizes that all theories of semantics must account for one essential feature of human language before such theories can begin to account for the analysis of thought encoding: speakers can understand (and produce) an infinite set of sentences.

Another route to answer this question is to put it differently: What must a native speaker know to understand a sentence? A popular view among linguists is that a native speaker needs to know two things to interpret a sentence: (1) the meaning of the component words of a sentence, and (2) the structure of the sentence. According to Bolhuis et al. (2014), hierarchical syntax is at the core of this complex cognitive system, language. Therefore, they take this component as the basic distinction between human language and any other non-human communication system. In their words, "...there is no evidence for human-like language syntax in any nonhuman species" (P. 2).

A third principle necessary for understanding a sentence is a set of finite rules that constrain the meaning of a sentence from ( $1 \& 2$ ) above (Partee 1995; Heim \&Kratzer 1998; Chierchia \& McConnellGinet 2000; Pagin \& Westerståhl 2010a, among others). Partee (1995, 2) states that this "fundamental aspect of semantic competence provides an argument that meanings must be governed by some version of the Principle of Compositionality," which essentially states that the semantic meaning of a complex utterance is determined by, or is a function of, the combined meanings of its components, that is, words and morphemes (Hintikka, 1984). Partee $(1995,2)$ suggests that "the principle of compositionality requires a notion of part-whole structure that is based on syntactic structure." Because of this, Partee (1995: 2) posits a revised form of semantic compositionality: "The meaning of a whole is a function of the meanings of the parts and of the way they are syntactically combined". Zadrozny $(1994,330)$ proposes that compositional semantics "intuitively" means that "we assign semantics to parse [syntactic] trees and not to strings of words." Pustejovsky and Boguraev (1993) put forth an analysis of compositionality that states that all lexical meanings in a phrase contribute to the meaning of a phrase overall. What all this means is that compositionality is "crucial to the process of semantic interpretation," a view which is different from some traditional account of semantics where verbs are lexical functions and nouns are lexical arguments of the functions (Pustejovsky and Boguraev, 1993: 202)).

Given the popular view in the available contemporary semantics literature, we could say that phrases have compositional meanings, but the words (phrasal constituents) themselves have extensions and intensions. Frege (1892) proposed that a word or expression has a "reference" and 
"sense", a line of demarcation which is akin to the modern distinctions of extension and intension. The extension or referent of a phrase is a truth-value (either true or false) and the intension is a thought or proposition (Frege 1892 in Neale 1999, 36), which, as we characterize it, is a function from possible worlds to truth-values of a phrase in those worlds.

For Frege (1892), the reason one can understand a sentence is not necessarily that he or she has heard it before. Rather, one understands a sentence because he or she knows the meanings of some basic components and the significance of combining those components in different ways. For example, before a human speaker of English can understand the meaning of 'The dogs like ice cream', they must first understand the meanings of the, $d o g$, -s, like, ice, and cream. Once they have conceptualized these constituents, they can determine the functions of the phrase from possible worlds to the truth-values of the phrase in each world. If in this world, dogs do like ice cream, then the truth-value is 'true'.

A complication of semantic meaning in human communication, however, is that phonological similarity does not entail a semantic similarity and vice versa. For instance, 'The dogs hate ice cream' is phonologically identical to 'The dogs like ice cream' except for the use of the word hate, but the semantic meaning of this phrase is the direct opposite. Likewise, two paraphrases of one semantic meaning may be vastly phonologically different, yet they could communicate similar meanings. It is also important to consider that phonological similarity does not entail semantic similarity (and vice versa) (see our discussion on the existence of semantic compositionality in animal communication systems above). Given the literature on the topic, we would expect that if two calls or cries in, for instance, Bonobo monkeys are phonologically similar, then they would have a similar semantic meaning. In other words, the monkey would never use one phrase of a cry that means "danger" in another cry meant, for example, to convey "happiness".

Zadrozny (1994:330) offers a summary of what we want semantics to be able to explain in modern language when stating that we essentially "want semantics to encode some basic intuitions, e.g. that nouns denote sets of entities, and verbs denote relations between entities, and so on; in other words, we would like to have a compositional semantics that agrees with intuitions". Also, no matter what we decide a sentence means semantically, it is "always possible to produce a function that would give compositional semantics to it" (Ibid, 330). Such a semantic compositional function in human language could be stated as follows (simplified from Zadrozny 1994):

$\mu(\mathrm{x} . \mathrm{y})=\mu(\mathrm{x})(\mu(\mathrm{y})) \quad$ Function $\mu$ with this definition gives us the compositional semantics for $\mathrm{S}$, a set of expressions, for all its elements (x.y) which are composed by concatenation of elements $\mathrm{x}$ and y.

The process of semantics in language production, then, could be thought of as follows: the conventional argument structures encode the mapping from a particular word to a particular semantic function, and then the syntactic realization of that word is related with the syntactic realizations of the other words in a phrase. After this has been done for all the constituents in a phrase, the syntactic structure as a whole is then parsed by compositional semantics (Pustejovsky and Boguraev 1993; Zadrozny 1994). It is important to note at this point that if we were to say that the communication system of some animals possessed the feature of compositional semantics as defined and described here, it would be to subsume that not only were the animals capable of associating one constituent with one semantic meaning but that they were also able to apply a semantic function (such as that of $\mu$ given above) to the meaning that would then relate it to all the other meanings of the other constituents that they produced or intercepted.

\section{Conclusions}

Throughout the long journey from a pre-linguistic state to full modern language, compositionality has played a substantial role in making this journey possible. Its highly effective role aided in putting human language one big step ahead. This compositional state was a springboard to the emergence of hierarchical syntax, hence the emergence of a recursive, fully complex language. On the other hand, non-human animal communication systems, including those examined in this paper, have not 
managed to progress beyond the holophrastic state, and thus remained highly confined and unproductive. The majority of these systems utilize mostly innate, function-limited unparsed holophrases. In other words, these systems are deemed context-restricted and event-related as their utterances being very limited in scope, size, and function. Despite this, only very few animal communication systems have been found to be combinatorial, thus bearing a modicum of truth towards compositionality. This is interesting in the study of language evolution as it could suggest that human language could not have evolved from a rudimentary stage called protolanguage. Speculating about the existence of protolanguage subsumes convergent evolution (e.g. songbirds) which has been shown untenable. The lack of semantic compositionality in non-human communication suggests that convergent evolution is hard to prove, which puts protolanguage on the line. This thesis is, however, far from being established and requires a lot of further research to prove its validity.

\section{References}

Arbib, Michael A. 2002. "The Mirror System, Imitation, and the Evolution of Language." Imitation in animals and artifacts 229 .

Arbib, Michael A. 2003. "The evolving mirror system: a neural basis for language readiness." Studies in the Evolution of Language 3: 182-200.

Arbib, Michael A. 2008. "Holophrasis and the protolanguage spectrum." Interaction Studies 9, no. 1: 154-168.

Arbib, Michael A., KatjaLiebal, Simone Pika, Michael C. Corballis, Chris Knight, David A. Leavens, Dario Maestripieri et al. 2008."Primate vocalization, gesture, and the evolution of human language." Current anthropology 49, no. 6: 1053-1076.

Arbib, Michael A., and Derek Bickerton, eds. 2010. The emergence of protolanguage: Holophrasisvs compositionality. Vol. 24. John Benjamins Publishing.

Arbib, M.A., 2017. Toward the language-ready brain: biological evolution and primate comparisons. Psychonomic bulletin \& review, 24(1), pp.142-150.

Berwick, R. C., \& Chomsky, N. (2011). The biolinguistic program: The current state of its development. The biolinguistic enterprise: New perspectives on the evolution and nature of the human language faculty, 19-41.

Berwick, Robert C., Angela D. Friederici, Noam Chomsky, and Johan J. Bolhuis. "Evolution, brain, and the nature of language. 2013." Trends in cognitive sciences 17, no. 2: 89-98.

Bickerton, Derek. 1990. Language and species. University of Chicago Press.

Bickerton, Derek. 1995. Language and human behavior. University of Washington Press.

Bickerton, D., 2002. From protolanguage to language. The speciation of modern Homo sapiens, pp.103-120.

Bickerton, Derek. 2007. "Language evolution: A brief guide for linguists." Linguai17, no. 3: 510-526.

Bickerton, Derek. 2010. "But how did protolanguage actually start?" The Emergence of Protolanguage: HolophrasisVs Compositionality 24: 167.

Bolhuis, Johan J., Ian Tattersall, Noam Chomsky, and Robert C. Berwick.2014. "How could language have evolved?" PLoS biology 12, no. 8: e1001934.

Buss, David M., Martie G. Haselton, Todd K. Shackelford, April L. Bleske, and Jerome C. Wakefield.1998."Adaptations, exaptations, and spandrels." American psychologist 53, no. 5: 533.

Chierchia, Gennaro, and Sally McConnell-Ginet. 200o. Meaning and grammar: An introduction to semantics. MIT press.

Chomsky, N. 1972. $\neg$ Language and Mind, 2nd edition. New York: Harcourt, Brace \&Javanovich.

Chomsky, N. (2010). Some simple evo devo theses: How true might they be for language. The evolution of human language: Biolinguistic perspectives, 62, 54-62.

Chomsky, N., 2017. Language architecture and its import for evolution. Neuroscience \& Biobehavioral Reviews, 81, pp.295-300.

Freeberg, Todd M., and Jeffrey R. Lucas. 2002. "Receivers respond differently to chick- a-dee calls varying in note composition in Carolina chickadees, Poecile carolinensis." Animal Behaviour 63, no. 5: 837-845.

Frege, Gottlob. 1892. "Über Sinn und Bedeutung." ZeitschriftfürPhilosophie und PhilosophischeKritik 100, 25-550. ("On sense and reference" in P. T. Geach and M. Black, eds. 1980.Translations from the Philosophical Writings of GottlobFrege. 56-78. Oxford: Blackwell.

Hauser, Marc D., Noam Chomsky, and W. Tecumseh Fitch. 2002. "The faculty of language: what is it, who has it, and how did it evolve?." science 298, no. 5598: 1569-1579.

Kratzer, Angelika, and Irene Heim. 1998. Semantics in generative grammar. Blackwell. 
Hintikka, J. 1984. "A hundred years later: The rise and fall of Frege's influence in language theory." Synthese 59, 2749.

Hockett, C. F. 1960. "The origin of speech. Human communication: language and its psychobiological bases. Readings from Scientific American."

Hurford, J. 2008. "The evolution of human communication and language." Sociobiology of communication: an interdisciplinary perspective. 249-264.

Jackendoff, Ray. 1999. "Possible stages in the evolution of the language capacity." Trends in cognitive sciences 3, no. 7: 272-279.

Jackendoff, Ray .2002. Foundations of language. Oxford, UK: Oxford University Press.

Jackendoff, Ray. 2011. "What is the human language faculty? Two views." Language 87, no. 3 :586-624.

Neale, Stephen. 1999. "Colouring and composition." In Philosophy and Linguistics, 35- 82. Westview Press.

Nóbrega, V.A. and Miyagawa, S., 2015. The precedence of syntax in the rapid emergence of human language in evolution as defined by the integration hypothesis. Frontiers in psychology, 6, p.271.

Miyagawa, S., Lesure, C. and Nóbrega, V.A., 2018. Cross-modality information transfer: a hypothesis about the relationship among prehistoric cave paintings, symbolic thinking, and the emergence of language. Frontiers in psychology, 9, p.115.

Pagin, Peter, and Dag Westerståhl.2010a. "Compositionality I: Definitions and variants." Philosophy Compass 5, no. 3: 250-264.

Partee, Barbara. 1995. "Lexical semantics and compositionality." An invitation to c ognitive science: Language 1: $311-$ 360.

Pepperberg, I. M. 1999. The Alex Studies. Cambridge, Massachusetts: Harvard University Press.

Pinker, steven. 1994. "The language instinct." How the Mind Creates Language. NY.

Pinker, Steven. 2003. "Language as an adaptation to the cognitive niche." Studies in the Evolution of Language 3: 1637.

Pustejovsky, James and BranimirBoguraev. 1993. "Lexical knowledge representation and natural language processing." Artificial Intelligence 63, 193-223.

Robinson, John G. 1984. "Syntactic structures in the vocalizations of wedge-capped capuchin monkeys, Cebusolivaceus." Behaviour 90:1, 46-79, August 1984.

Smith, Kenny. 2006. "The protolanguage debate: bridging the gap." Cangelosi et al. 315-322.

Smith, Kenny. 2008. "Is a holistic protolanguage a plausible precursor to language? A test case for a modern evolutionary linguistics." Interaction Studies 9, no. 1: 1- 17.

Schel, A. M., Tranquilli, S. and Zuberbühler, K. 2009. "The alarm call system of two species of black-and-white colobus monkeys (Colobuspolykomos and Colobusguereza)." Journal of Comparative Psychology 123.2: 136150 .

Tallerman, M. 2007. "Did our ancestors speak a holistic protolanguage?" Lingua 117.3: 579-604.

Tallerman, M. 2010. Holophrastic protolanguage: Planning, processing, storage, and retrieval. In Arbib\&Bickerton (eds.), 83-97.

Tallerman, M., 2016. Against the emergent view of language evolution. Proceedings of EvoLang XI.

Tallerman, M., 2017. Can the integration hypothesis account for language evolution? Journal of Neurolinguistics, 43, pp.254-262.

Wray, Alison. 2002a. "Dual processing in protolanguage: performance without competence." 113-137.

Szathmáry, Eörs, and John Maynard Smith. 1995. "The major evolutionary transitions." Nature 374, no. 6519: 227232.

Zadrozny, Wlodek. 1994. "From compositional to systematic semantics." Linguistics and Philosophy 17, 329-342.

Zuberbühler, Klaus. 2005."Linguistic prerequisites in the primate lineage." In Language origins: Perspectives on evolution, pp. 262-282. Oxford University Press. 